\title{
APLICAÇÃO DE UV LED NO ENSAIO POR PARTÍCULAS MAGNÉTICAS NA LINHA DE INSPEÇÃO DE TARUGOS DA GERDAU OURO BRANCO*
}

\section{Resumo}

\author{
Guilherme Lisboa Quaresma ${ }^{1}$ \\ Alexsandro Elson Teixeira ${ }^{2}$ \\ Luiz Orione Vieira ${ }^{3}$ \\ Antonio Marcos Venanzi ${ }^{4}$ \\ Adriano Schirmer Silva ${ }^{5}$ \\ Marcio Renato Da Silva ${ }^{6}$ \\ Edimar da Consolação Santos ${ }^{7}$
}

Um ensaio por partículas magnéticas é um método não destrutivo utilizado na detecção de descontinuidades superficiais e subsuperficiais em produtos laminados. Este método consiste em submeter a peça a um campo magnético, com aglomeração de partículas magnetizáveis nos pontos onde há descontinuidade. As partículas usadas podem ser de diferentes cores, dimensões e formas. A partícula fluorescente sob a luz ultravioleta demonstra melhor resultado para detecção de pequenas descontinuidades. Para ampliar o campo de visão ensaiado e realçar o contraste entre partícula e peça a radiação ultravioleta deve garantir uniformidade, intensidade e comprimento de onda adequado. Essas exigências motivaram o trabalho da Gerdau junto com a Metal Chek, representante da Spectroline, no desenvolvimento de uma solução inovadora. Na Linha de Inspeção de Tarugos da Gerdau Ouro Branco o uso de luminárias ultravioletas de LED - Light Emitting Diode tornou-se uma alternativa que proporcionou ganho em eficiência e em confiabilidade na tomada de decisão do inspetor de qualidade, baixo consumo de energia, custo de manutenção e satisfação dos clientes. A implantação das luminárias de LED refletiu na prática a evolução da qualidade do produto, além de melhores condições em meio ambiente, saúde e segurança para o inspetor.

Palavras-chave: Partícula magnética fluorescente; Luz ultravioleta; LED - Light Emitting Diode; Qualidade.

\section{USING UV LED IN MAGNETIC PARTICLE TESTING IN THE LINE OF BILLETS INSPECTION OF GERDAU OURO BRANCO}

\begin{abstract}
A magnetic particle testing is a non-destructive method for detecting discontinuities in surfaces and subsurfaces in laminated products. This method consists of submiting the product to a magnetic field, with an agglomeration of magnetizable particles at the points where there is discontinuity. The particles used may have different colors, sizes and shapes. The fluorescent particle under ultraviolet light shows a better result for the detection of small discontinuities. To expand the field of view tested and in order to highlight the contrast between particle and item, the ultraviolet radiation must ensure uniformity, intensity and appropriate wavelength. These requirements motivated the work of Gerdau along with Metal Chek, representant of Spectroline, in the development of an innovative solution. In the Line of Billet Inspection of Gerdau Ouro Branco, the usage of ultraviolet lamps of LED - Light Emitting Diode, became an alternative that provided efficiency and reliability gains in the decision making process of the quality inspector, low power consumption, cost maintenance and customer satisfaction. The introduction of LED lamps reflected in the evolution of product quality, better environmental conditions, health and safety to the inspector.

Keywords: Fluorescent magnetic particle; Ultraviolet light; LED - Light Emitting Diode; Quality.

Engenheiro de Produção, Chefe Leitos e Pontes, Laminações de Longos, Gerdau, Ouro Branco, MG, Brasil.

Eng. de Minas, Chefe Inspeção e Acabamento, Laminações de Longos, Gerdau, Ouro Branco, MG, Brasil.

Técnico Mecânico, Assessor Técnico, Laminações de Longos, Gerdau, Ouro Branco, MG, Brasil.

Técnico Metalurgista, Supervisor Técnico, Metal Chek, Brança Paulista, SP, Brasil.

Engenheiro Mecânico, Vendedor Técnico, Metal Chek, Belo Horizonte, MG, Brasil.

Eng. Eletricista, M.Sc. Técnico eletricista, Laminação e Acabamento PBT, Gerdau, Ouro Branco, MG, Brasil.

Técnico em Metalurgia, Inspetor de Qualidade, Laminações de Longos, Gerdau, Ouro Branco, MG, Brasil.
\end{abstract}

* Contribuição técnica ao $51^{\circ}$ Seminário de Laminação - Processos e Produtos Laminados e Revestidos, 28 a 31 de outubro de 2014, Foz do Iguaçu, PR, Brasil. 


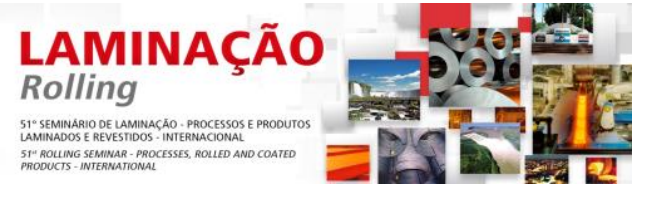

Tabela 2. Comprimento de onda por espectro e cor

\begin{tabular}{|l|l|c|}
\hline Espectro & \multicolumn{1}{|c|}{ Cor } & $\boldsymbol{\lambda}(\mathbf{n m})$ \\
\hline \multirow{4}{*}{ Ultravioleta } & $\mathrm{C}$ & $180-280$ \\
\cline { 2 - 3 } & $\mathrm{B}$ & $280-315$ \\
\cline { 2 - 3 } & $\mathrm{A}$ & $315-400$ \\
\hline \multirow{4}{*}{ Luz visível } & Violeta & $400-440$ \\
\cline { 2 - 3 } & Azul & $440-485$ \\
\cline { 2 - 3 } & Ciano & $485-500$ \\
\cline { 2 - 3 } & Verde & $500-565$ \\
\cline { 2 - 3 } & Amarelo & $565-590$ \\
\cline { 2 - 3 } & Laranja & $590-625$ \\
\cline { 2 - 3 } & Vermelho & $625-740$ \\
\hline Infravermelho & ABC & $740-1 \times 10^{6}$ \\
\hline
\end{tabular}

Para a escolha da luminária ideal a ser utilizada no ensaio como fonte da energia eletromagnética que estimulará a florescência da partícula, objetivamos a emissão monocromática na faixa UVA, no comprimento de onda de $365 \mathrm{~nm}$ e ainda atingir a intensidade conforme indicado pela norma ASTM E 1444 (valor mínimo de 1000 $\mu \mathrm{W} / \mathrm{cm}^{2}$ na superfície ensaiada). Comumente utilizam-se lâmpadas de vapor de mercúrio para alcançar essas condições de iluminação. Essas lâmpadas se baseiam no princípio da descarga elétrica por meio de gases. Porém as buscas por melhores resultados de eficiência na intensidade luminosa e exatidão no comprimento de onda tem motivado o surgimento de alternativas viáveis. Daí, a tecnologia LED (Light Emitting Diode, ou Diodo Emissor de Luz) se transformou em um dos principais alvos de avanços na área de iluminação, alinhando conforto visual, flexibilidade de aplicação e uso, eficiência energética e sustentabilidade.

O diodo emissor de luz LED é um componente eletrônico semicondutor que tem a propriedade de transformar energia elétrica em luz na matéria, diferente das tecnologias convencionais que necessitam de descarga de gases, filamentos metálicos, radiação ultravioleta e outros. Para obter o uso dessa tecnologia no ensaio de partículas magnéticas é necessário encontrar um diodo capaz de emitir um comprimento de onda de $365 \mathrm{~nm}$ pelos motivos explicamos acima.

\section{MATERIAIS E MÉTODOS}

A linha de inspeção de tarugos (LIT) da Gerdau Ouro Branco possui equipamento de inspeção superficial com método de partículas magnéticas por via úmida, utilizando concentrado a base de água com pigmentação fluorescente na cor amarelo intenso. Para criar o campo magnético que tenha força suficiente de direcionar as partículas para os campos de fuga gerados nas descontinuidades, utilizamos duas estações de magnetização composta por bobinas transversais e longitudinais que promovem uma magnetização multidirecional por indução. A peça é ensaiada em rota continua de processo, na posição diamante em dois postos de inspeção com iluminação luz ultravioleta (ou "luz negra") na superfície do material.

\footnotetext{
* Contribuição técnica ao $51^{\circ}$ Seminário de Laminação - Processos e Produtos Laminados e Revestidos, 28 a 31 de outubro de 2014, Foz do Iguaçu, PR, Brasil.
} 

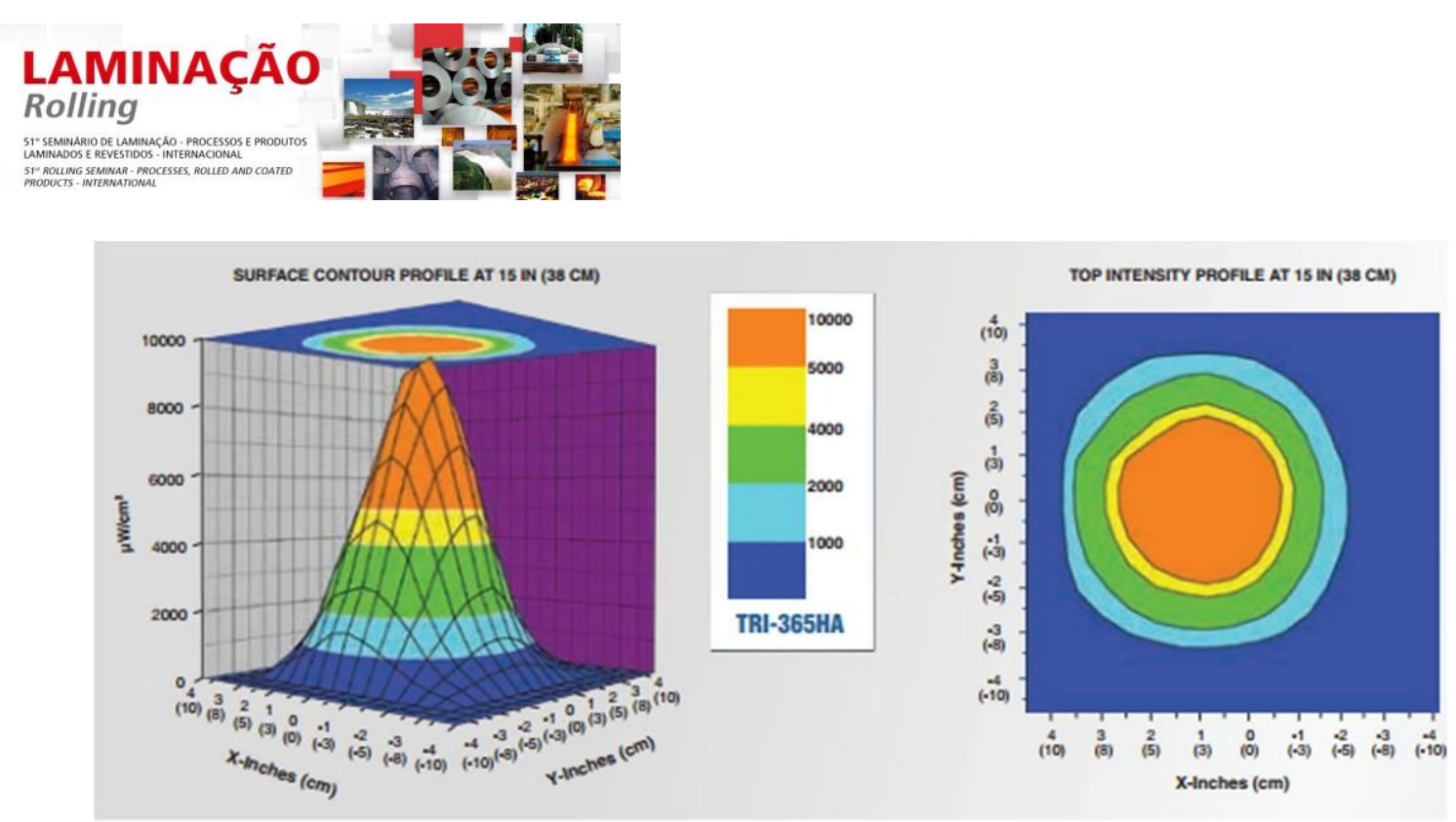

Figura 3 - Modelo de intensidade e foco da luminária portátil de LED

A partir deste resultado propusemos a criação de uma luminária com essa tecnologia, porém com dimensões que atendessem a necessidade da máquina de inspeção da Gerdau Ouro Branco.

Foi desenvolvido então um modelo utilizando os LED's da luminária Tritan dispostos em linha. Chegamos a uma dimensão de $40 \mathrm{~cm}$ de comprimento, com $15 \mathrm{~cm}$ de altura, mudanças no circuito elétrico e ventilação, a fim de atender ao campo visual do inspetor e a instalação no local de trabalho. Em função desse trabalho foi criado o modelo comercial PowerMax 365. Esta luminária somou a característica da alta intensidade, ampliação da região de inspeção e diodos emitindo radiação no comprimento de onda em torno do desejado de $365 \mathrm{~nm}$.

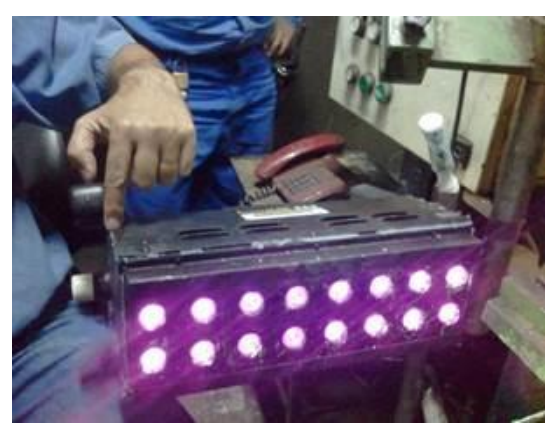

Figura 4. Protótipo inicial

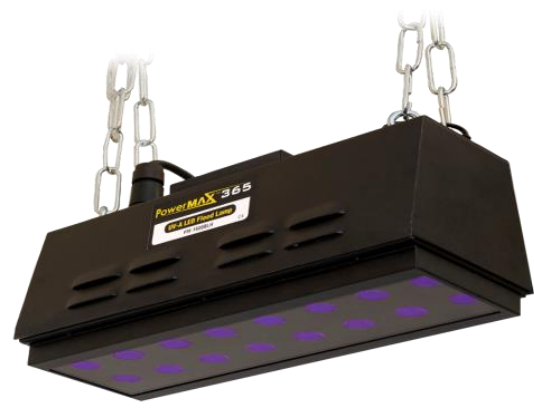

Figura 5. Modelo comercial

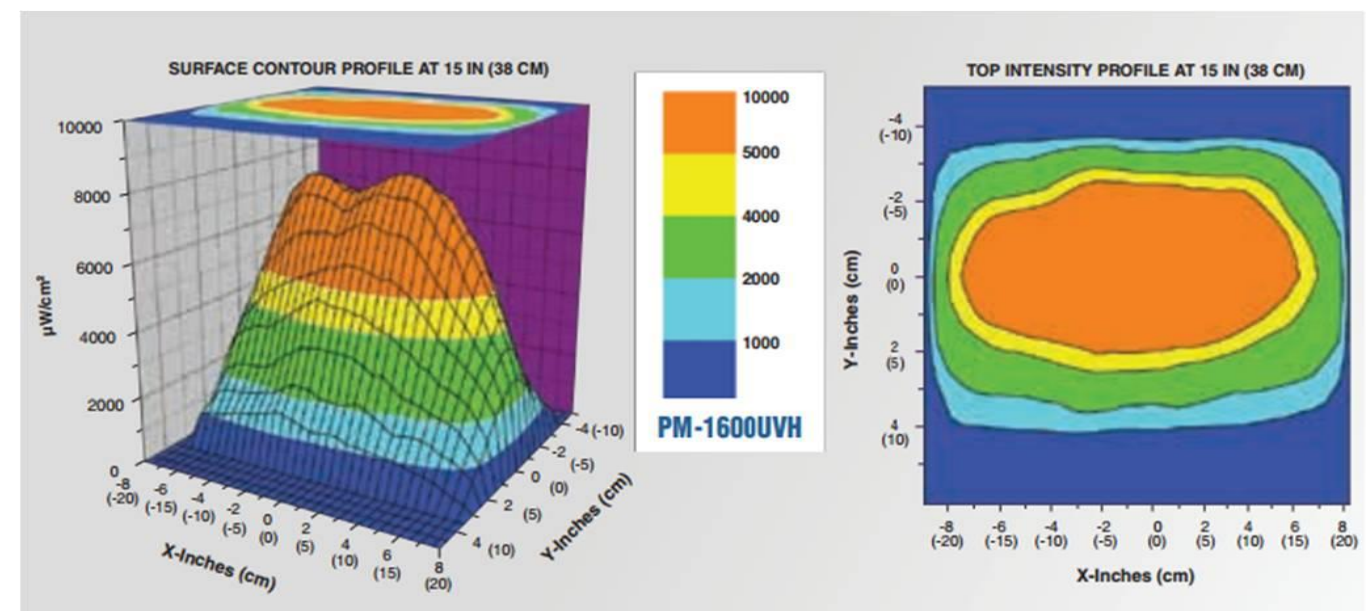

Figura 6 - Modelo de intensidade e foco da luminária de LED desenvolvida

* Contribuição técnica ao $51^{\circ}$ Seminário de Laminação - Processos e Produtos Laminados e Revestidos, 28 a 31 de outubro de 2014, Foz do Iguaçu, PR, Brasil. 


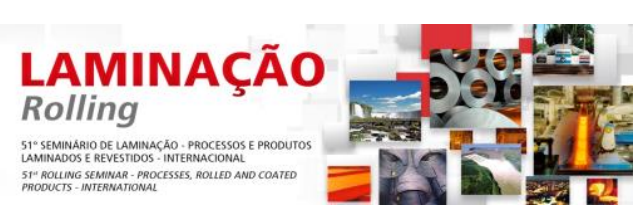

Na figura 7, a curva PM-1600UV representa a distribuição dos resultados de comprimento de onda, obtida pela emissão dos diodos da luminária desenvolvida.

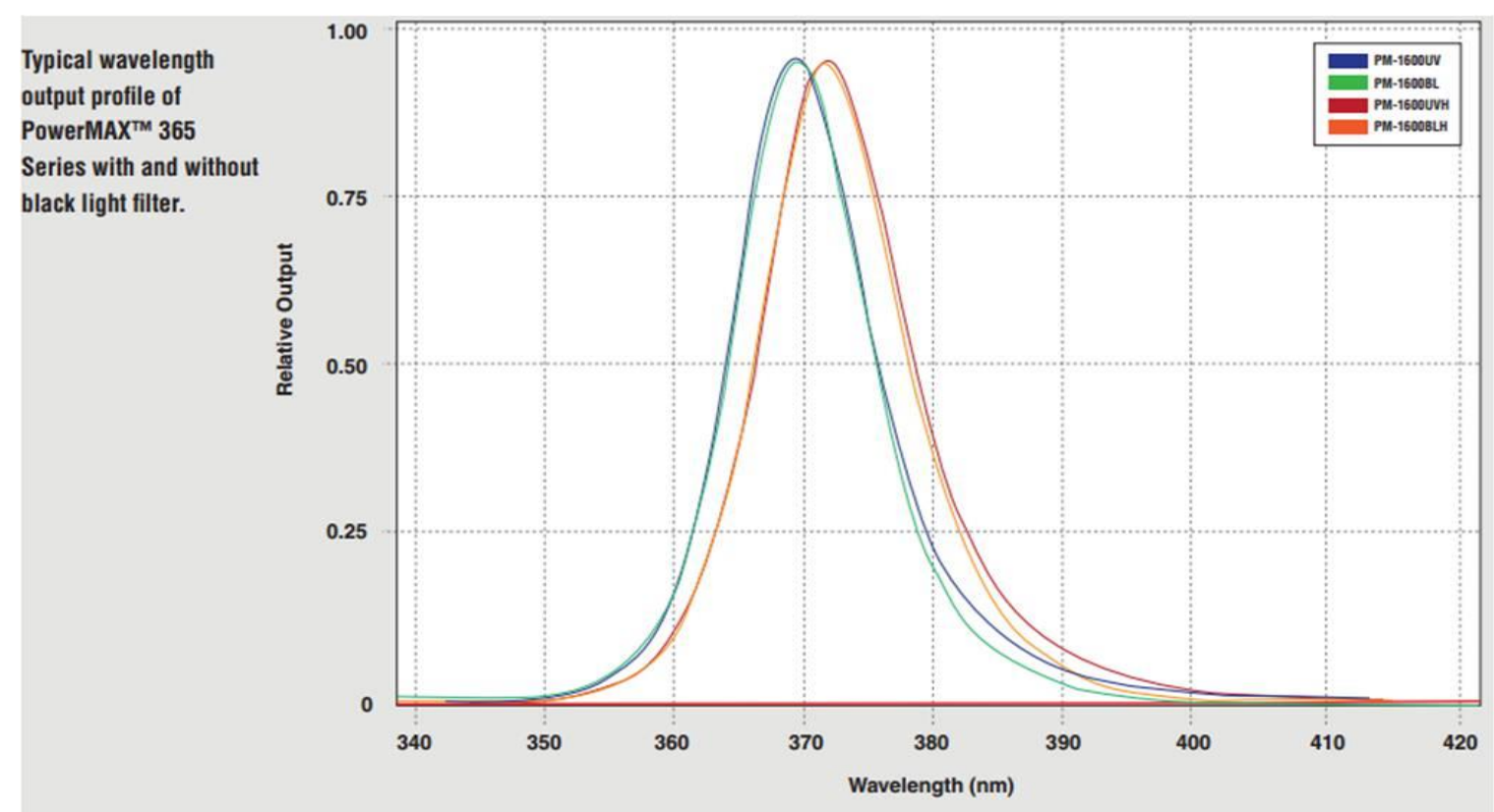

Figura 7 - Distribuição normalizada dos comprimentos de onda da luminária de LED desenvolvida.

\section{RESULTADOS E DISCUSSÃO}

Um dos defeitos superficiais comuns em produtos laminados são as trincas longitudinais. Uma forma de remover estes defeitos é o esmerilhamento. Por procedimento interno quando o produto apresenta como resultado da inspeção nível $D$ de defeitos, ou seja, mais de $70 \%$ de sua superfície, este produto será reinspecionado visando garantir a identificação de todos os defeitos relevantes.

Após a implementação do trabalho foi possível obter redução de $83 \%$ no número de defeitos superficiais encontrados em etapas de reinspeção das peças. Este resultado foi possível devido à ampliação da região com luminosidade adequada para o ensaio e maior florescência nas descontinuidades reveladas (em função da precisão no comprimento de onda em torno de $365 \mathrm{~nm}$, mostrado na figura 7 ).

Redução no consumo de energia, aumento da vida útil das luminárias, maior resistência á impactos mecânicos e vibrações, versatilidade no layout de instalação, não aquecimento da estrutura da luminária eliminando o risco de queimaduras, sustentabilidade por não conter metais pesados como mercúrio são outras vantagens obtidas pela tecnologia LED.

\section{CONCLUSÃO}

Para manter a preferência dos clientes é fundamental garantir no final do processo, produtos que atendam na íntegra as exigências mais altas do mercado. A etapa de inspeção de qualidade requer parâmetros bem controlados, monitoramentos periódicos e níveis de confiabilidade que assegurem uma elevada assertividade na tomada de decisão. O trabalho demonstrou que a atualização tecnológica pode elevar esses níveis de resultado e contribuir para eficiência da relação custo/benefício. 
A iluminação LED ultravioleta possibilitou avanços claros nas condições do ensaio de partículas magnéticas. $O$ ganho no aumento da florescência das descontinuidades oferece condições para implantação de um sistema automatizado, através de reconhecimento de imagens. O aumento da região em condições de ser ensaiada abriu espaço para o ganho de produtividade na inspeção.

\section{Agradecimentos}

Os autores agradecem pelo suporte, disponibilização de recursos e dedicação de toda a equipe envolvida na parceria entre Metal Chek, Spectroline e Gerdau que proporcionaram a realização deste trabalho.

\section{REFERÊNCIAS}

1 Andreucci R. Partículas Magnéticas, 2009 - Publicação da Associação Brasileira de Ensaios Não Destrutivos e Inspeção.

2 Turro NJ. Modern molecular photochemistry, 1a edição, University Science Books, 1991.

* Contribuição técnica ao $51^{\circ}$ Seminário de Laminação - Processos e Produtos Laminados e Revestidos, 28 a 31 de outubro de 2014, Foz do Iguaçu, PR, Brasil. 